\title{
Exact quantum dynamics in structured environments
}

\author{
Dominic Gribben $\odot,{ }^{1, *}$ Aidan Strathearn,,${ }^{1,2}$ Jake Iles-Smith, ${ }^{3}$ Dainius Kilda,,${ }^{1, \dagger}$ Ahsan Nazir $\odot,{ }^{4}$ \\ Brendon W. Lovett, ${ }^{1, \$}$ and Peter Kirton $\odot^{5, \S}$ \\ ${ }^{1}$ SUPA, School of Physics and Astronomy, University of St Andrews, St Andrews KY16 9SS, United Kingdom \\ ${ }^{2}$ School of Mathematics and Physics, The University of Queensland, St Lucia, Queensland 4072, Australia \\ ${ }^{3}$ Department of Physics and Astronomy, University of Sheffield, Sheffield S3 7RH, United Kingdom \\ ${ }^{4}$ Department of Physics and Astronomy, The University of Manchester, Oxford Road, Manchester M13 9PL, United Kingdom \\ ${ }^{5}$ Vienna Center for Quantum Science and Technology, Atominstitut, TU Wien, 1040 Vienna, Austria
}

(Received 12 July 2019; accepted 31 January 2020; published 5 March 2020)

\begin{abstract}
The dynamics of a wide range of technologically important quantum systems are dominated by their interaction with just a few environmental modes. Such highly structured environments give rise to long-lived bath correlations that induce complex dynamics which are very difficult to simulate. These difficulties are further aggravated when spatial correlations between different parts of the system are important. By modeling the dynamics of a pair of two-level quantum systems in a common, structured, environment we show that a recently developed general purpose numerical approach, the time-evolving matrix product operator, is capable of accurate simulation under exactly these conditions. We find that tuning the separation to match the wavelength of the dominant environmental modes can drastically modify the system dynamics. To further explore this behavior, we show that the full dynamics of the bath can be calculated directly from those of the system, thus allowing us to develop intuition for the complex dynamics observed.
\end{abstract}

DOI: 10.1103/PhysRevResearch.2.013265

\section{INTRODUCTION}

When a spatially extended quantum system interacts with a structured environment in which a narrow band of modes dominate, the resulting dynamics can be very complex and difficult to simulate accurately. Such environments can retain a memory of their interactions with the system on a time scale comparable to that on which the state of the system changes, and in the face of such memory effects standard open system techniques can fail. However, reliable simulation of such environments is vital in order to understand the behavior of an ever-increasing number of experimental platforms and quantum devices. For example, in photosynthetic systems the observation of quantum coherence arises from the interplay of electronic coupling to groups of vibrational modes [1] and the coupling between biomolecules [2]. The interplay of these degrees of freedom has been shown to enhance energy transport [3-6], where spatial correlations in the environment

\footnotetext{
*dg99@st-andrews.ac.uk

†Present address: Division of Chemistry and Chemical Engineering, California Institute of Technology, Pasadena, CA, 91125, USA.

‡bw14@st-andrews.ac.uk

${ }^{\S}$ Present address: Department of Physics and SUPA, University of Strathclyde, Glasgow G4 0NG, United Kingdom; peter.kirton@ strath.ac.uk
}

Published by the American Physical Society under the terms of the Creative Commons Attribution 4.0 International license. Further distribution of this work must maintain attribution to the author(s) and the published article's title, journal citation, and DOI. play a crucial role [7-11]. Similar physics can also be found in, e.g., trapped ions [12] and molecular junctions [13].

The sensitivity of extended quantum systems to memory effects was recently observed in an experiment on a "giant" superconducting atom [14], and spatially correlated noise in quantum registers has been shown to affect quantum error propagation [15]. Bath memory also plays a key role in the function of micromechanical resonators, where adjustment of the environmental noise spectrum is possible [16] and photonic crystals, where band gaps in the spectral density give rise to localized modes and dissipationless oscillations [17-19]. The effect of structured environments has also been explored with superconducting qubits subject to noise or varying bandwidth [20].

In order to model these environmental memory effects, we must go beyond the standard Born-Markov approximations [21] which lead to time-local master equations for the system density matrix. The development of techniques to simulate these non-Markovian dynamics has therefore been the subject of much recent theoretical effort [22]. These techniques broadly fall into two categories: First, there are approximate methods which change the boundary between the system and environment such that the Born-Markov approximations are valid for the new system [23-28]; second, there are numerically exact methods which utilize some particular structure of the bath Hamiltonian to provide exact dynamics [29-35]. These approaches usually work best for models where the system Hilbert space is quite small, or has a specific form of spectral density. However, it can often be difficult to know a priori the range of validity of some of these approaches, and so accurate and efficient benchmarking procedures are essential. 


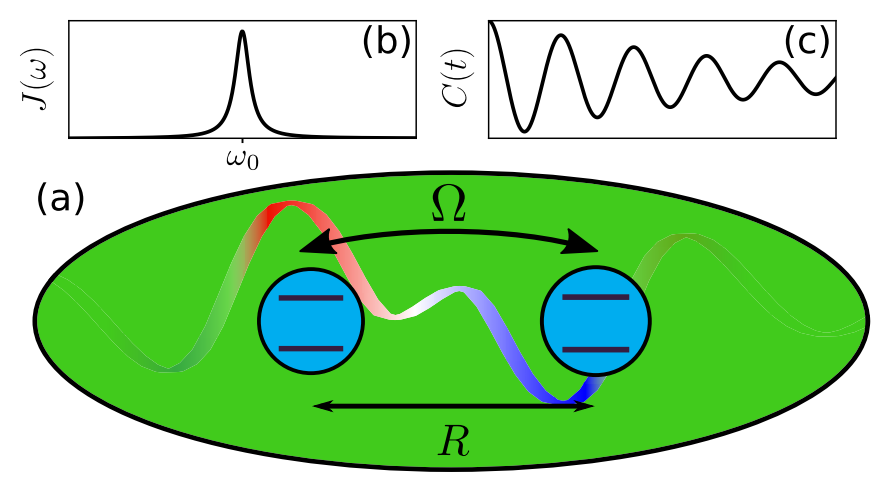

FIG. 1. (a) Cartoon of the dimer system we consider accompanied by schematic plots of (b) the underdamped spectral density $J(\omega)$ and (c) the corresponding bath autocorrelation function $C(t)$.

In this paper, we demonstrate that a recently developed numerically exact approach, the time-evolving matrix product operator (TEMPO) [34], can be used to efficiently find accurate nonlocal system dynamics for highly structured environments including spatial effects. The TEMPO algorithm uses a Feynman sum-over-histories approach to the pathintegral formulation of the dynamics of an open quantum system, using an influence functional to capture environmental memory effects. By writing this path-integral representation as a sum over discrete time steps, we can calculate the system quantum dynamics by contracting a tensor network [34]. The memory effects are then encoded in a matrix product state which contains information about the history of the system. This is propagated forward in time by successive contraction with a matrix product operator. By performing singular value decompositions and truncation after each time step [36,37] we can capture bath memory times orders-of-magnitude beyond those possible with previous approaches [30,31]. The similarities between TEMPO and the process tensor [38] have recently been explored [39], and TEMPO has been used to model dynamics in optomechanical systems [40]. Further details on TEMPO can be found in Appendix A.

We use TEMPO to study a pair of spatially separated twolevel subsystems interacting with a common environment, in which a narrow band of modes dominate the interaction. The complex environmental structure and spatial correlations give rise to highly non-Markovian system dynamics and we find that by tuning the separation between the subsystems, it is possible to control their interaction with the environment. We also show how we may obtain the dynamics of the environment by appropriate analysis of purely system observables. This then allows us to develop a more complete picture of the complex quantum dynamics of this model using TEMPO, going beyond the calculation of expectation values of system operators that is typically done using exact approaches.

\section{MODEL}

Figure 1(a) shows a schematic cartoon of the model we consider and highlights how the pair of two-level systems in- teract both directly and via the environment. The Hamiltonian for this model is given by

$$
\begin{aligned}
H= & \sum_{i} \epsilon_{i}\left|X_{i}\right\rangle\left\langle X_{i}\right|+\frac{\Omega}{2}\left(\left|X_{1}\right\rangle\left\langle X_{2}|+| X_{2}\right\rangle\left\langle X_{1}\right|\right) \\
& +\sum_{i} \sigma_{z, i} \sum_{k}\left(g_{i, k} a_{k}+g_{i, k}^{*} a_{k}^{\dagger}\right)+\sum_{k} \omega_{k} a_{k}^{\dagger} a_{k} .
\end{aligned}
$$

The two-level system (TLS) at site $i$ and position $r_{i}$ has energy splitting $\epsilon_{i}$ and an excited (ground) state denoted $\left|X_{i}\right\rangle\left(\left|0_{i}\right\rangle\right)$. The pair of TLSs form a dimer with a coherent coupling of strength $\Omega$. The environment consists of bosons confined to one dimension where $a_{k}^{\dagger}$ creates an excitation in the mode with wave vector $k$. We also introduce the Pauli operators $\sigma_{z, i}=\left|X_{i}\right\rangle\left\langle X_{i}|-| 0_{i}\right\rangle\left\langle 0_{i}\right|$. By assuming that the environment is uniform then the coupling $g_{i, k}=g_{k} \exp \left(-i k r_{i}\right)$ consists of a position independent part $g_{k}$ and a phase that depends on the site $i$. This kind of model can underpin a wide range of physical systems, for example biological or molecular systems undergoing energy transport and interacting with vibrational modes [5,41], energy transfer in solid-state systems [42], superconducting qubits in microwave resonators [43], or quantum dots interacting with a micromechanical resonator [44].

The bath can be completely characterized by its spectral density $J(\omega)=\sum_{k}\left|g_{k}\right|^{2} \delta\left(\omega-\omega_{k}\right)$, from which we can calculate its autocorrelation function in thermal equilibrium at temperature $T$ :

$$
C(t)=\int_{0}^{\infty} d \omega J(\omega)\left[\operatorname{coth}\left(\frac{\omega}{2 T}\right) \cos (\omega t)-i \sin (\omega t)\right] .
$$

If this function decays slowly compared to the system time scales then the bath memory is important.

We consider a bath in which a narrow band of modes dominate the interaction with the system. The TEMPO method is not limited to studying such forms of spectral density; it has already been well tested for relatively flat functional forms $[34,40]$-instead the long-lived correlations provide a challenge for the method to capture. The specific form of spectral density we use is [45]

$$
J_{0}(\omega)=\frac{\alpha \Gamma \omega_{0}^{2} \omega}{\left(\omega_{0}^{2}-\omega^{2}\right)^{2}+\Gamma^{2} \omega^{2}} .
$$

Here $\alpha$ gives the coupling strength, $\omega_{0}$ is the frequency characterizing the dominant mode, and $\Gamma$ provides a measure of the width of the dominant band. Figure 1(b) shows a schematic plot of this spectral density and Fig. 1(c) the corresponding correlation function highlighting the long memory times present with such environments.

The Hamiltonian, Eq. (1), does not couple states with a different total number of system excitations and so we restrict ourselves to the single excitation subspace, the only one where nontrivial dynamics are observed. Within this subspace it is possible to map the problem onto that of a single spin coupled to a bosonic environment [46]. The mapped Hamiltonian is then a spin-boson Hamiltonian,

$$
H=\frac{\Omega}{2} \sigma_{x}+\frac{\epsilon}{2} \sigma_{z}+\sigma_{z} \sum_{k}\left(\tilde{g}_{k} a_{k}+\tilde{g}_{k}^{*} a_{k}^{\dagger}\right)+\sum_{k} \omega_{k} a_{k}^{\dagger} a_{k},
$$




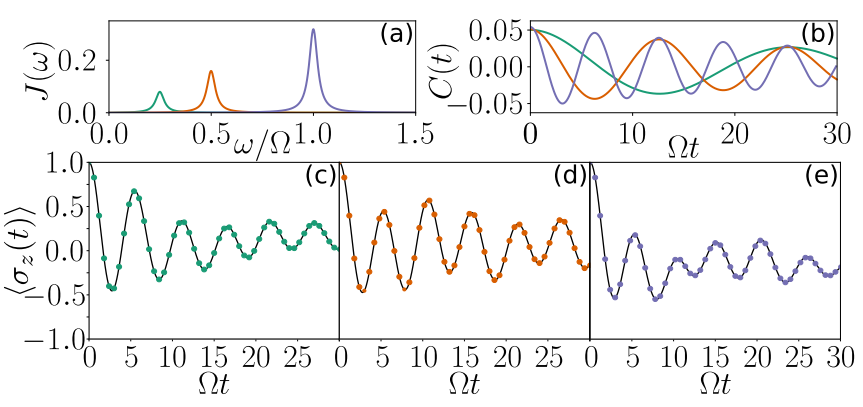

FIG. 2. (a) Underdamped spectral density, given by Eq. (3), with the different frequencies used. (b) Corresponding correlation functions. (c)-(e) Dynamics calculated using TEMPO (dots) and $\mathrm{RC}$ (solid lines) for $\omega_{0}=\Omega / 4, \Omega / 2, \Omega$. The other parameters are $\pi \alpha=0.05 \Omega, \Gamma=0.05 \Omega, T=\Omega, \epsilon=0.5 \Omega$.

where $\epsilon=\epsilon_{2}-\epsilon_{1}$ and $\tilde{g}_{k}=2 i g_{k} \sin \left[k\left(r_{1}-r_{2}\right) / 2\right]$. We also define new Pauli operators: $\sigma_{z}=\left(\sigma_{z, 2}-\sigma_{z, 1}\right) / 2$ and $\sigma_{x}=$ $\left|X_{2}\right\rangle\left\langle X_{1}|+| X_{1}\right\rangle\left\langle X_{2}\right|$. This modification to the coupling constants results in a renormalization of the spectral density,

$$
J(\omega)=2 J_{0}(\omega)[1-\cos (\omega R)],
$$

where $R=\left|r_{1}-r_{2}\right|$ is the dimer separation and we have assumed a linear dispersion $\omega(k)=c|k|$ with $c=1$. The cosine term arises from the phase factors in the original coupling terms.

\section{BENCHMARKING}

In order to check that TEMPO remains effective for highly structured environments we first benchmark it for a simplified case of a single spin described by Eq. (4) interacting with a bath with the unmodified spectral density, Eq. (3). In this limit it is possible to simulate the dynamics using other techniques. In particular we compare the TEMPO simulations with those obtained using a reaction-coordinate (RC) master equation, which has been rigorously benchmarked for similar problems [26-28], allowing us to verify that TEMPO is able to accurately simulate dynamics in structured environments. The $\mathrm{RC}$ mapping takes a single collective mode of the environment into the system definition; then the rest of the bath, which is assumed to be Markovian, couples to the now augmented system. This gives rise to a standard Born-Markov master equation for the system dynamics. More details can be found in Appendix B.

In Fig. 2 we compare dynamics generated by TEMPO with those of the RC master equation for this simplified model, testing three different values of $\omega_{0}$. We find excellent agreement between the two algorithms for all sets of parameters. The $\mathrm{RC}$ approach is accurate for these parameters and this form of spectral density, and so is able to capture the complex dimer dynamics. In this simple case the RC approach requires significantly reduced numerical resources compared to TEMPO, though we find that both techniques take longer to converge at lower mode frequencies and higher temperatures. For the $\mathrm{RC}$ algorithm the reason for this is clear: the occupation of the mode included in the system increases in these regimes and so a larger system Hilbert space is needed for convergence. For TEMPO we find that the number of singular values that need
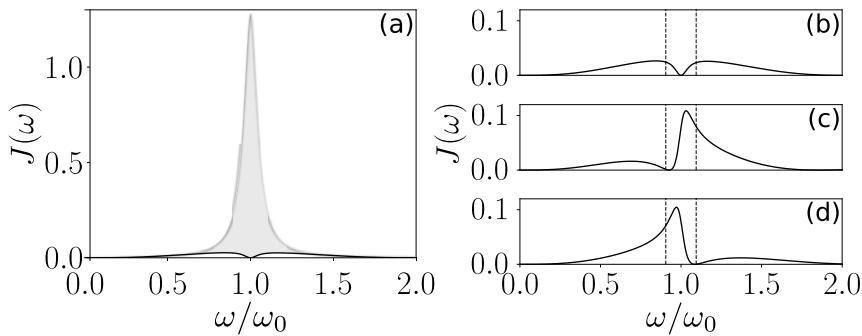

FIG. 3. Effect of the mapping, Eq. (5), on the spectral density. (a) The unmapped spectral density (grey shaded) and an example of the mapped case (solid black). (b)-(d) Mapped spectral densities for the three cases considered in the main text from top to bottom: $R=2 \pi / \omega_{0}, 2 \pi / 1.1 \omega_{0}$, and $2 \pi / 0.9 \omega_{0}$. The dashed black lines depict the frequencies described in the main text.

to be retained for convergence increases-i.e., more system paths gain a significant amplitude. This is expected when the environment becomes more occupied. These results show both that TEMPO is able to deal with such structured spectral densities and the RC approach can be used to accurately simulate dynamics for this model [28].

\section{EXACT SYSTEM DYNAMICS}

We now turn to analyzing the full model including the spatial modifications to the spectral density present in Eq. (5). Previous studies have shown that spatial correlations in the bath can have a significant effect on population transfer between localized systems [7-9,34]. However, these studies focused on the case where the original spectral density $J_{0}(\omega)$ is much broader than that given in Eq. (3) - they do not include systems where just a narrow band of modes dominate the system interaction - and so the modification due to the spatial structure of the system is not as pronounced.

Since we are now using the general mapped spectral density in Eq. (5), treating the dynamics using the RC formalism becomes much more difficult: to accurately capture the dynamics it would be necessary to include more modes in the system than is computationally feasible. This means that for the remainder of this paper all results are obtained using TEMPO.

In this model there are now three possible resonance conditions which can be met by matching two of the following: the bare system frequency $\Omega$, the characteristic frequency of the environment $\omega_{0}$, and the frequency corresponding to the separation between the TLSs $\omega_{R}=2 \pi / R$. In Fig. 3 we show how choosing $\omega_{R}=\omega_{0}$ leads to a complete suppression of the main peak of the bare spectral density, and choosing $\omega_{R}=(1 \pm 0.1) \omega_{0}$ leads to a very asymmetric line shape. In the single line plots of Fig. 4 we show how these differences in spectral density manifest themselves in the system dynamics. We show results for the three TLS spacings $\omega_{R}=\omega_{0},(1 \pm$ $0.1) \omega_{0}$ and for bare system frequencies $\Omega$ tuned to the same three possible values, resulting in nine sets of results.

The system dynamics are complex and difficult to interpret, with multiple oscillation frequencies appearing in the dynamics. These are not only due to the peaked structure of the original spectral density (as occurred for the simplified 


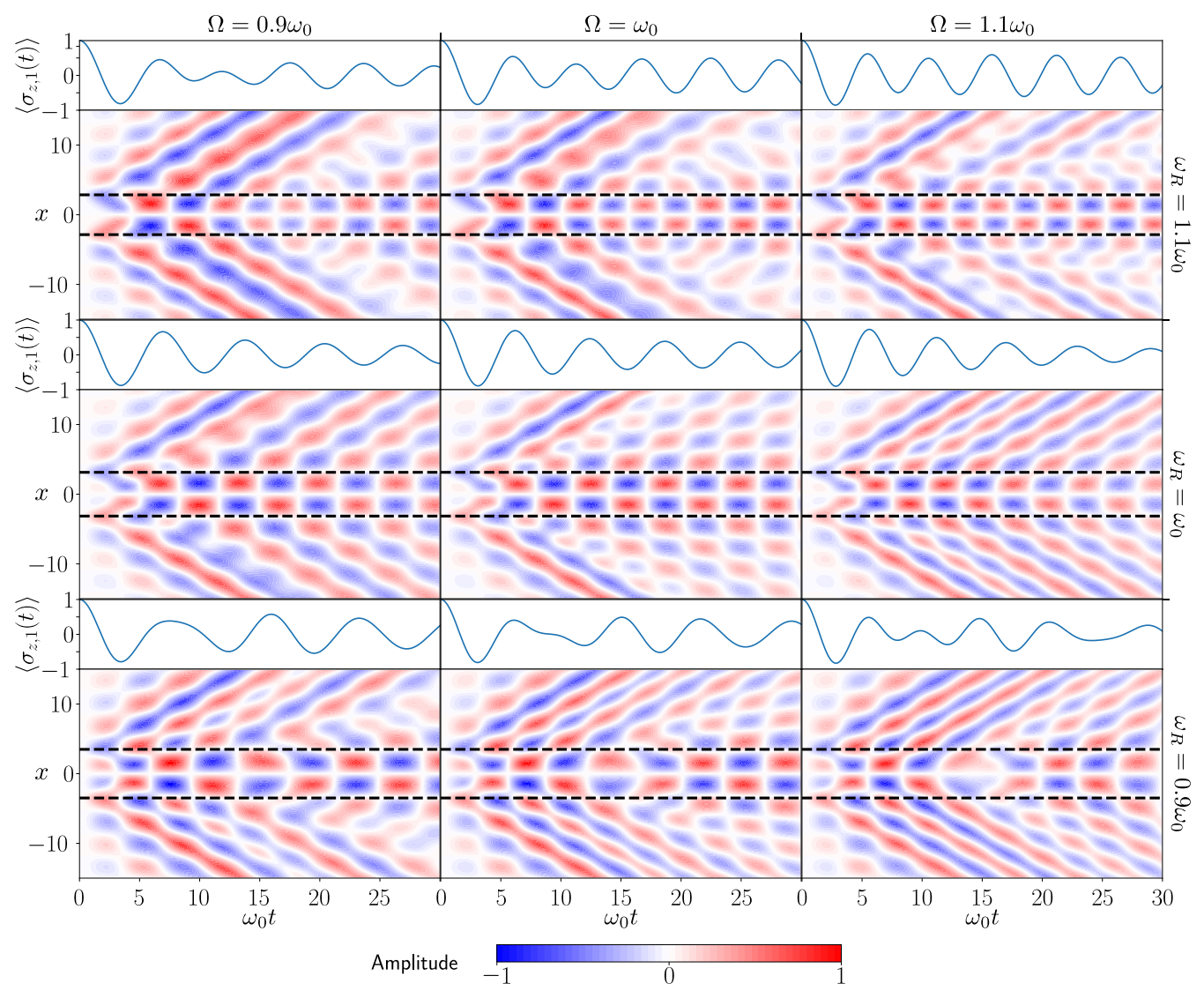

FIG. 4. Spin dynamics (single line plots) and corresponding environment displacement (main panels) at position $x$ over time as calculated with Eq. (16) for various separations and dimer frequencies. Displacements are normalized to the maximum displacement across all plots. The dashed black lines correspond to the positions of the TLSs. For all of the dynamics we have set $T=0$ and $\epsilon=0, \Gamma=0.05 \omega_{0}$, and $\pi \alpha=0.1 \omega_{0}$ for the unmapped spectral density.

single spin model) but also because of the spatial correlations. However, we next show that TEMPO can be used not only to extract system dynamics, as discussed in Ref. [34], but also to find evolution of bath degrees of freedom. This will enable us to gain more insight into how the interplay between the bath and system leads to the complex dynamics shown in Fig. 4.

\section{BATH DYNAMICS}

In this section we show that it is possible to calculate bath operator expectation values in terms of system dynamics. To do this we formally solve the Heisenberg equations of motion $[47,48]$ for the expectation value of, e.g., the annihilation operator for a particular bath mode, and obtain an expression entirely in terms of the expectation of the system operator which couples to the bath.

Consider general interaction and bath Hamiltonians given by

$$
H_{I}(t)+H_{B}=\hat{A}(t) \sum_{k}\left(g_{k} a_{k}^{\dagger}+g_{k}^{*} a_{k}\right)+\sum_{k} \omega_{k} a_{k}^{\dagger} a_{k},
$$

for a system operator $\hat{A}(t)$ in the system interaction picture. The time evolution of the annihilation operator $a_{k}(t)$ can be found from the Heisenberg equation of motion:

$$
\begin{aligned}
\frac{d}{d t} a_{k}(t) & =i\left[H_{I}(t)+H_{B}, a_{k}(t)\right] \\
& =-i g_{k} \hat{A}(t)-i \omega_{k} a_{k}(t), \\
\frac{d}{d t}\left[e^{i \omega_{k} t} a_{k}(t)\right] & =-i g_{k} e^{i \omega_{k} t} \hat{A}(t) .
\end{aligned}
$$

Formally integrating this and taking the expectation with respect to the total density matrix $\rho$ gives (assuming $\left.\left\langle a_{k}(0)\right\rangle=0\right)$

$$
\begin{gathered}
\left\langle a_{k}(t)\right\rangle=-i g_{k} e^{-i \omega_{k} t} \int_{0}^{t} d t^{\prime} e^{i \omega_{k} t^{\prime}}\left\langle\hat{A}\left(t^{\prime}\right)\right\rangle, \\
\left\langle a_{k}^{\dagger}(t)\right\rangle=i g_{k}^{*} e^{i \omega_{k} t} \int_{0}^{t} d t^{\prime} e^{-i \omega_{k} t^{\prime}}\left\langle\hat{A}\left(t^{\prime}\right)\right\rangle .
\end{gathered}
$$

Thus the dynamics of any bath mode can be found from knowing the exact system dynamics up to the time $t$. Calculations of higher-order moments are much more involved requiring multiple numerical integrals to be performed and so for now we only consider quantities that can be constructed from the expressions above.

The quantity we consider is the displacement of a onedimensional field [49] given by

$$
\Phi(x, t)=\sum_{k} \frac{1}{\sqrt{\omega_{k}}}\left[\left\langle a_{k}(t)\right\rangle+\left\langle a_{-k}^{\dagger}(t)\right\rangle\right] e^{i k x} .
$$


Now for the $\hat{A}=\sigma_{z}$ coupling from Eq. (4) we have $\tilde{g}_{-k}^{*}=\tilde{g}_{k}$ and $\omega_{k}=|k|=\omega_{-k}$ such that

$$
\begin{aligned}
\Phi(x, t) & =\sum_{k} \frac{i \tilde{g}_{k}}{\sqrt{\omega_{k}}}\left[-e^{-i \omega_{k} t} \int_{0}^{t} d t^{\prime} e^{i \omega_{k} t^{\prime}}\left\langle\sigma_{z}\left(t^{\prime}\right)\right\rangle+e^{i \omega_{k} t} \int_{0}^{t} d t^{\prime} e^{-i \omega_{k} t^{\prime}}\left\langle\sigma_{z}\left(t^{\prime}\right)\right\rangle\right] e^{i k x} \\
& =-\sum_{k} \frac{2 \tilde{g}_{k}}{\sqrt{\omega_{k}}} e^{i k x} \int_{0}^{t} d t^{\prime} \sin \left[\omega_{k}\left(t-t^{\prime}\right)\right]\left\langle\sigma_{z}\left(t^{\prime}\right)\right\rangle \\
& =-4 i \int_{-\infty}^{\infty} d k \frac{g_{k}}{\sqrt{\omega_{k}}} \sin \left(\frac{k R}{2}\right) e^{i k x} \int_{0}^{t} d t^{\prime} \sin \left[\omega_{k}\left(t-t^{\prime}\right)\right]\left\langle\sigma_{z}\left(t^{\prime}\right)\right\rangle \\
& =8 \int_{0}^{\infty} d \omega \sqrt{\frac{J_{0}(\omega)}{\omega}} \sin \left(\frac{\omega R}{2}\right) \sin (\omega x) \int_{0}^{t} d t^{\prime} \sin \left[\omega\left(t-t^{\prime}\right)\right]\left\langle\sigma_{z}\left(t^{\prime}\right)\right\rangle,
\end{aligned}
$$

where between the last two lines we have discarded the odd component of the integrand and halved the integration domain since everything remaining is even.

We show the real-space displacement of the bath as a function of time and position as a color map beneath the corresponding system dynamics in Fig. 4. The black dotted lines indicate the positions of the two TLSs. In the fully resonant case (the center plot) we see that most of the bath excitation gets trapped between the two TLSs which simply show oscillations which decay away at a very slow rate. We also see slow decay in the other two plots where $\Omega=\omega_{R}$; this is because $J(\Omega)=0$ in these cases and hence a simple Markovian treatment would predict no decay at all.

When at least one of the frequencies is detuned from this condition we see a more pronounced propagation of bath excitations away from the system as the trapping effect is reduced. In these cases the overall decay rate of the TLS is gradually enhanced as the detuning is increased, since now the value of $J(\Omega)$ is larger. The detuning can also introduce a beat frequency into the bath dynamics, most clearly seen in the bath population trapped between the two TLSs, and most pronounced in the most detuned cases, i.e., in the bottom right and top left panels of Fig. 4. This beating gives rise to distinctive revivals in the TLS dynamics.

At very short times all of the dynamics are very similar. On time scales $t<\omega_{R}^{-1}$ each TLS only senses its own local environment and hence behaves as if it were interacting with bosons described by the unmapped spectral density. However as soon as the influence of the other TLS is felt the dynamics become radically different to those predicted by an independent environment model. These dynamics are highly sensitive to the relative detunings of the three frequencies described above, becoming significantly different even for the very small detunings studied here.

\section{CONCLUSION}

In conclusion, we have shown that TEMPO can provide accurate simulations of quantum systems in structured environments. We first validated the technique by checking that TEMPO simulations for a simple model agree with the RC approach. We then presented TEMPO simulations of the more complex dynamics that result from the interplay between a highly structured spectral function and spatial correlations between different parts of the system. This shows that TEMPO is not limited to specific forms of spectral density: it should be straightforward to go from single to multiple peaks, so long as the memory time of the bath can still be captured. TEMPO therefore provides a versatile method for simulating systems coupled to an environment with arbitrary structure.

In addition, we have shown that it is possible to find the full dynamics of the environment directly from the system dynamics. This enabled us to explore how it is possible to tune the separation between the spins such that one can effectively remove the coupling to the dominant environmental mode and how the resultant dynamics are highly sensitive to slight variations in parameters around this point. Such exact environmental tracking can help to explain the behavior of open quantum systems in general, and so aid in the design of future quantum devices. This greater insight into the dynamics of open quantum systems could also lead to the development of new approximation schemes and to the self-consistent verification of others.

The research data supporting this publication can be found in Ref. [50].

\section{ACKNOWLEDGMENTS}

We would like to thank J. Keeling for useful discussions. D.G. and D.K. acknowledge studentship funding from EPSRC under Grant No. EP/L015110/1. A.S. acknowledges a studentship from EPSRC under Grant No. EP/L505079/1. J.I.-S. acknowledges support from the Royal Commission for the Exhibition of 1851. A.N. acknowledges funding from EPSRC under Grant No. EP/N008154/1. P.K. acknowledges support from an ESQ fellowship of the Austrian Academy of Sciences (ÖAW).

\section{APPENDIX A: TIME-EVOLVING MATRIX PRODUCT OPERATORS}

In this Appendix we outline the implementation of the time-evolving matrix product operator (TEMPO) algorithm. For full details we refer the reader to Ref. [34].

The TEMPO algorithm is built upon the quasiadiabatic path-integral (QUAPI) method [30]. To calculate the nonMarkovian evolution of the system time is discretized using steps of size $\Delta t$. Then by assuming the system operators are constant over each time step the evolution up to time $t_{N}=N \Delta t$ may be calculated by summing over all possible 
paths the system has taken to that point from initial time $t_{0}$. In practice this can be done through an iterative tensor propagation routine where the object propagated is the augmented density tensor (ADT). The ADT is grown up to time $t_{N}$ from an initial physical density matrix $\rho_{i_{1}}\left(t_{1}\right)$ through iterative application of tensors:

$$
A^{j_{N} \ldots j_{1}}\left(t_{N}\right)=\prod_{n=2}^{N} B^{j_{n}, j_{n-1}, \ldots, j_{1}} \begin{gathered}
i_{n-1}, \ldots, i_{1} \\
i_{i_{1}}
\end{gathered}\left(t_{1}\right) .
$$

The form of the $B$ tensor depends only on the influence functional of the Gaussian bath and can be found in Ref. [34]. The reduced system density matrix at time $t_{N}$ is then given by summing over all indices except for the current time $\rho_{j_{N}}\left(t_{N}\right)=$ $\sum_{j_{1} \ldots j_{N-1}} A^{j_{N} \ldots j_{1}}\left(t_{N}\right)$. For a harmonic bath linearly coupled to the system, the $B$ tensor is composed of influences across all pairs of time points from $t_{1}$ to $t_{N}$, as well as time-local components due to the coherent Hamiltonian evolution. The discretization time step $\Delta t$ needs to both resolve the features of the correlation function and ensure that the Trotter errors are negligible. Without further considerations the exponential growth of the ADT only allows memory lengths of order $\sim 20 \Delta t$ to be simulated. This restricts QUAPI to baths with relatively short-lived and/or slowly varying autocorrelation functions.

In TEMPO the ADT is built in the form of a matrix product state (MPS) [36,37] and a singular value decomposition (SVD) sweep is carried out at each time step. The $B$ tensors are composed of a product of time-local operators and keeping these components separate leads to the natural formation of an MPS representation for the $A$ tensor given by

$$
A^{j_{N} \ldots j_{1}}\left(t_{N}\right)=\sum_{\alpha_{1} \ldots \alpha_{N}}\left[a^{j_{N}}\right]_{\alpha_{N}}\left[a^{j_{N-1}}\right]_{\alpha_{N}, \alpha_{N-1}} \ldots\left[a^{j_{1}}\right]_{\alpha_{1}} .
$$

where the tensors at the two ends of the chain are rank 2, and those in between are rank 3. In this notation the superscripts are the "physical" index which connects to the propagation tensor while the subscripts link adjoining $a$ tensors. At each step of the propagation an SVD is performed on each $a$ and singular values below a fixed precision $\chi$ (relative to the largest) are discarded. This leads to a reduced dimension of the internal indices $\alpha$ and a significant improvement on the exponential scaling present in QUAPI. The truncation acts to remove the least important internal degrees of freedom that are not needed for achieving converged dynamics. This procedure has been shown to reduce the scaling of the required computational resources to polynomial with memory length for smooth spectral densities [34].

In practice there are two convergence parameters associated with TEMPO which must be adjusted to produce the exact results in the main text:

(i) The size of the discretization time step $\Delta t$, decreased until convergence.

(ii) The singular value cutoff $\chi$ reduced until convergence. This is the ratio between the largest singular value and the smallest which we retain.

Further to these, with TEMPO a memory cutoff is also possible such that the growth of the ADT is stopped once it covers enough of the system's history to capture all relevant memory effects. For the results presented in the main
TABLE I. Convergence parameters used for figures in the main text.

\begin{tabular}{lcc}
\hline \hline Result & Time step $\Delta t$ & Cutoff $\chi$ \\
\hline Fig. 2 & $0.2 / \Omega$ & $10^{-8}$ \\
Fig. 4 & $0.1 / \omega_{0}$ & $10^{-7}$ \\
\hline
\end{tabular}

text, however, the long correlation times associated with the structured environments make this kind of memory cutoff impossible and as such none were used for generating any of the results. Table I gives the convergence parameters used for each of the results.

\section{APPENDIX B: REACTION COORDINATE MASTER EQUATION}

In this Appendix we outline how the reaction coordinate (RC) mapping is implemented to obtain a time-local master equation for the two-level system-RC (TLS-RC) reduced density matrix. The RC is the coordinate corresponding to the direction the bath equilibrium position is displaced when the system transitions between $\sigma_{z}$ eigenstates. It corresponds to a superposition of normal modes of the bath, and is defined by the following mapping:

$$
H_{I}=\sigma_{z} \sum_{k} g_{k}\left(a_{k}^{\dagger}+a_{k}\right) \rightarrow \lambda \sigma_{z}\left(c^{\dagger}+c\right) .
$$

Here, $c^{\dagger}(c)$ create (destroy) an excitation in the reaction coordinate mode. The form of the transformation results in coupling between the RC and the residual modes $b_{k}$. The spin-boson Hamiltonian is then mapped to the following form:

$$
\begin{gathered}
H_{\mathrm{RC}}=H_{S}+H_{I}+H_{B}+H_{C}, \\
H_{S}=\frac{\Omega}{2} \sigma_{x}+\frac{\epsilon}{2} \sigma_{z}+\lambda \sigma_{z}\left(c^{\dagger}+c\right)+\Omega_{\mathrm{RC}} c^{\dagger} c, \\
H_{I}=\left(c^{\dagger}+c\right) \sum_{k} d_{k}\left(b_{k}^{\dagger}+b_{k}\right), \\
H_{B}=\sum_{k} v_{k} b_{k}^{\dagger} b_{k}, \\
H_{C}=\left(c^{\dagger}+c\right)^{2} \sum_{k} \frac{d_{k}^{2}}{\omega_{k}},
\end{gathered}
$$

where the last term exists to counter a renormalization of the RC's potential that arises from the interaction term. Here the TLS-RC coupling is given by $\lambda^{2}=\sum_{k} g_{k}^{2}$ and this form ensures that the canonical bosonic commutation relation is satisfied. This coupling leads to an RC frequency of $\Omega_{\mathrm{RC}}=$ $\lambda^{-2} \sum_{k} \omega_{k} g_{k}^{2}$. The residual environment is characterized by a new spectral density $J_{\mathrm{RC}}(\omega)=\sum_{k}\left|d_{k}\right|^{2} \delta\left(\omega-\omega_{k}\right)$ which can be found by replacing the TLS in both the mapped and unmapped Hamiltonians with a classical coordinate [51]. The spectral density does not contain any information about the system itself and therefore can be found, in both cases, through the classical equations of motion of this coordinate $[26,52]$. The underdamped spectral density considered in the main text leads to $\Omega_{\mathrm{RC}}=\omega_{0}, \lambda=\sqrt{\pi \alpha_{\mathrm{UD}} \omega_{0} / 2}$ and $J_{\mathrm{RC}}=$ $\gamma \omega$ where $\gamma=\Gamma / 2 \pi \omega_{0}$. 
Once this mapping has been performed we follow the procedure outlined in Ref. [26] applying both Born and Markov approximations to arrive at the Schrödinger picture master equation for the reduced TLS-RC density operator $\rho(t)$ :

$$
\begin{aligned}
\frac{\partial \rho}{\partial t}= & -i\left[H_{S}, \rho(t)\right]-\gamma \int_{0}^{\infty} d \tau \int_{0}^{\infty} d \omega \omega \cos (\omega \tau) \operatorname{coth}\left(\frac{\omega}{2 T}\right) \\
& \times[\hat{C},[\hat{C}(-\tau), \rho(t)]]-\gamma \int_{0}^{\infty} d \tau \int_{0}^{\infty} d \omega \omega \cos (\omega \tau) \\
& \times\left[\hat{C},\left\{\left[\hat{C}(-\tau), H_{S}\right], \rho(t)\right\}\right],
\end{aligned}
$$

where we have defined $\hat{C}=c^{\dagger}+c$ and $\hat{C}(t)$ is the same operator in the interaction picture at time $t$. Truncating the Hilbert space of the RC down to $n$ basis states, i.e., permitting a maximum of only $n$ excitations, allows us to numerically diagonalize $H_{S}$. This gives us a set of basis states $\left|\phi_{j}\right\rangle$ which satisfy $H_{S}\left|\phi_{j}\right\rangle=\phi_{j}\left|\phi_{j}\right\rangle$ allowing us to express the interaction picture operators as

$$
\hat{C}(t)=\sum_{j, k=1}^{2 n} C_{j k} e^{i \omega_{j k} t}\left|\phi_{j}\right\rangle\left\langle\phi_{k}\right|,
$$

where $C_{j k}=\left\langle\phi_{j}|\hat{C}| \phi_{k}\right\rangle$ and $\omega_{j k}=\phi_{j}-\phi_{k}$. We can then rewrite Eq. (B7) as

$$
\frac{\partial \rho}{\partial t}=-i\left[H_{S}, \rho(t)\right]-[\hat{\boldsymbol{C}},[\hat{\chi}, \rho(t)]]+[\hat{\boldsymbol{C}},\{\hat{\boldsymbol{E}}, \rho(t)\}]
$$

with rate operators:

$$
\begin{gathered}
\hat{\chi}=\frac{\pi}{2} \sum_{j k} J_{\mathrm{RC}}\left(\xi_{j k}\right) \operatorname{coth}\left(\frac{\xi_{j k}}{2 T}\right) C_{j k}\left|\phi_{j}\right\rangle\left\langle\phi_{k}\right|, \\
\hat{\Xi}=\frac{\pi}{2} \sum_{j k} J_{\mathrm{RC}}\left(\xi_{j k}\right) C_{j k}\left|\phi_{j}\right\rangle\left\langle\phi_{k}\right| .
\end{gathered}
$$

The resulting master equation can then be solved using standard open systems approaches for Markovian systems [21].
[1] J. Adolphs and T. Renger, Biophys. J. 91, 2778 (2006).

[2] D. B. Turner, K. E. Wilk, P. M. G. Curmi, and G. D. Scholes, J. Phys. Chem. Lett. 2, 1904 (2011).

[3] M. Thorwart, J. Eckel, J. Reina, P. Nalbach, and S. Weiss, Chem. Phys. Lett. 478, 234 (2009).

[4] A. W. Chin, J. Prior, R. Rosenbach, F. Caycedo-Soler, S. F. Huelga, and M. B. Plenio, Nat. Phys. 9, 113 (2013).

[5] M. del Rey, A. W. Chin, S. F. Huelga, and M. B. Plenio, J. Phys. Chem. Lett. 4, 903 (2013).

[6] M. Mohseni, A. Shabani, S. Lloyd, and H. Rabitz, J. Chem. Phys. 140, 035102 (2014).

[7] P. Nalbach, J. Eckel, and M. Thorwart, New J. Phys. 12, 065043 (2010).

[8] A. Nazir, Phys. Rev. Lett. 103, 146404 (2009).

[9] D. P. S. McCutcheon and A. Nazir, Phys. Rev. B 83, 165101 (2011)

[10] D. Abramavicius and S. Mukamel, J. Chem. Phys. 134, 174504 (2011).

[11] M. Sarovar, Y.-C. Cheng, and K. B. Whaley, Phys. Rev. E 83, 011906 (2011).

[12] C. Maier, T. Brydges, P. Jurcevic, N. Trautmann, C. Hempel, B. P. Lanyon, P. Hauke, R. Blatt, and C. F. Roos, Phys. Rev. Lett. 122, 050501 (2019).

[13] D. Segal and B. K. Agarwalla, Ann. Rev. Phys. Chem. 67, 185 (2016).

[14] G. Andersson, B. Suri, L. Guo, T. Aref, and P. Delsing, Nat. Phys. 15, 1123 (2019).

[15] D. Aharonov, A. Kitaev, and J. Preskill, Phys. Rev. Lett. 96, 050504 (2006).

[16] S. Gröblacher, A. Trubarov, N. Prigge, G. D. Cole, M. Aspelmeyer, and J. Eisert, Nat. Commun. 6, 7606 (2015).

[17] W.-M. Zhang, P.-Y. Lo, H.-N. Xiong, M. W.-Y. Tu, and F. Nori, Phys. Rev. Lett. 109, 170402 (2012).

[18] A. Goban, C.-L. Hung, S.-P. Yu, J. Hood, J. Muniz, J. Lee, M. Martin, A. McClung, K. Choi, D. Chang, O. Painter, and H. Kimble, Nat. Commun. 5, 3808 (2014).

[19] N. M. Sundaresan, R. Lundgren, G. Zhu, A. V. Gorshkov, and A. A. Houck, Phys. Rev. X 9, 011021 (2019).
[20] A. Potočnik, A. Bargerbos, F. A. Y. N. Schröder, S. A. Khan, M. C. Collodo, S. Gasparinetti, Y. Salathé, C. Creatore, C. Eichler, H. E. Türeci, A. W. Chin, and A. Wallraff, Nat. Commun. 9, 904 (2018).

[21] H.-P. Breuer and F. Petruccione, The Theory of Open Quantum Systems (Oxford University Press, Oxford, 2002).

[22] I. de Vega and D. Alonso, Rev. Mod. Phys. 89, 015001 (2017).

[23] R. Silbey and R. A. Harris, J. Chem. Phys. 80, 2615 (1984).

[24] R. A. Harris and R. Silbey, J. Chem. Phys. 83, 1069 (1985).

[25] S. Jang, Y.-C. Cheng, D. R. Reichman, and J. D. Eaves, J. Chem. Phys. 129, 101104 (2008).

[26] J. Iles-Smith, N. Lambert, and A. Nazir, Phys. Rev. A 90, 032114 (2014).

[27] J. Iles-Smith, Ph.D. thesis, Imperial College London, 2015.

[28] J. Iles-Smith, A. G. Dijkstra, N. Lambert, and A. Nazir, J. Chem. Phys. 144, 044110 (2016).

[29] Y. Tanimura and R. Kubo, J. Phys. Soc. Jpn. 58, 101 (1989).

[30] N. Makri and D. E. Makarov, J. Chem. Phys. 102, 4600 (1995).

[31] N. Makri and D. E. Makarov, J. Chem. Phys. 102, 4611 (1995).

[32] J. Prior, A. W. Chin, S. F. Huelga, and M. B. Plenio, Phys. Rev. Lett. 105, 050404 (2010).

[33] D. Suess, A. Eisfeld, and W. T. Strunz, Phys. Rev. Lett. 113, 150403 (2014).

[34] A. Strathearn, P. Kirton, D. Kilda, J. Keeling, and B. W. Lovett, Nat. Commun. 9, 3322 (2018).

[35] F. Mascherpa, A. Smirne, A. D. Somoza, P. Fernández-Acebal, S. Donadi, D. Tamascelli, S. F. Huelga, and M. B. Plenio, arXiv: 1904.04822.

[36] R. Orús, Ann. Phys. 349, 117 (2014).

[37] U. Schollwöck, Ann. Phys. 326, 96 (2011).

[38] F. A. Pollock, C. Rodríguez-Rosario, T. Frauenheim, M. Paternostro, and K. Modi, Phys. Rev. A 97, 012127 (2018).

[39] M. R. Jørgensen and F. A. Pollock, Phys. Rev. Lett. 123, 240602 (2019).

[40] Y. Minoguchi, P. Kirton, and P. Rabl, arXiv:1904.02164.

[41] S. Rackovsky and R. Silbey, Mol. Phys. 25, 61 (1973). 
[42] E. Rozbicki and P. Machnikowski, Phys. Rev. Lett. 100, 027401 (2008).

[43] H. Zheng and H. U. Baranger, Phys. Rev. Lett. 110, 113601 (2013).

[44] I. Yeo, P.-L. de Assis, A. Gloppe, E. Dupont-Ferrier, P. Verlot, N. S. Malik, E. Dupuy, J. Claudon, J.-M. Gérard, A. Auffèves, G. Nogues, S. Seidelin, J.-P. Poizat, O. Arcizet, and M. Richard, Nat. Nanotechnol. 9, 106 (2013).

[45] S. Mukamel, Principles of Nonlinear Optical Spectroscopy (Oxford University Press, New York, 1995), Vol. 29.

[46] T. M. Stace, A. C. Doherty, and S. D. Barrett, Phys. Rev. Lett. 95, 106801 (2005).

[47] C. W. Gardiner and M. J. Collett, Phys. Rev. A 31, 3761 (1985).
[48] C. Gardiner and P. Zoller, Quantum Noise: A Handbook of Markovian and Non-Markovian Quantum Stochastic Methods with Applications to Quantum Optics, Vol. 56 (Springer, New York, 2004).

[49] G. D. Mahan, Many-particle Physics (Springer, New York, 2013).

[50] D. Gribben, A. Strathearn, J. Iles-Smith, D. Kilda, A. Nazir, B. W. Lovett, and P. Kirton, Exact quantum dynamics in structured environments, https://doi.org/10.17630/36eff292-773a40b7-a8bc-9ec916191c9b (2020).

[51] A. Garg, J. N. Onuchic, and V. Ambegaokar, J. Chem. Phys. 83, 4491 (1985).

[52] A. J. Leggett, Phys. Rev. B 30, 1208 (1984). 